\title{
Schneller Digitaldruck von Lacken
}

\author{
Erstmals erlaubt eine neue Technologie zum schnellen Druck \\ von Lacken vielfältige Möglichkeiten für den Digitaldruck in \\ Produktionsverfahren. Die Technik könnte auch zukünftig bei der \\ automatisierten Serienproduktion zum Einsatz kommen.
}

$V_{\mathrm{i}}$ iele Hersteller versuchen zurzeit, ihre Unternehmen auf Industrie 4.0 auszurichten. Sie stehen dabei vor der komplexen Herausforderung, bisherige, oft noch manuelle Herstellprozesse auf automatisierte digitale Systeme umzurüsten. Covestro, ein führender Anbieter von Lack- und Klebstoffrohstoffen, und TTP, ein Technologieunternehmen im Bereich Digitaldruck, haben einen Durchbruch beim Digitaldruck von Lacken und PolyurethanKlebstoffen in Industriequalität erzielt. Die Technik könnte das Potenzial haben, die Umrüstung auf automatisierte Serienproduktion zu ermöglichen.

Die Kombination aus TTPs neuer Technologie für einen schnellen, digitalen Druck von Lacken und Klebstoffen und Covestros maßgeschneiderten Polyurethan-Rohstoffen eröffnet neue Möglichkeiten in Produktionsverfahren der nächsten Generation. „Erst kürzlich haben wir Klebstoffe auf Basis von Covestro-Rohstoffen erfolgreich getestet“, sagt Dr. Andrew Baker-Campbell, Projektleiter bei TTP. „Damit haben wir gezeigt, dass wir Materialien drucken können, die eine deutlich größere Herausforderung darstellen als herkömmliche InkjetTinten.“

\section{Neues Labor für Digitaldruck}

Um die Entwicklung voranzutreiben, hat Covestro ein eigenes Labor für Digitaldruck eingerichtet. „Der Digitaldruck von Lacken und Klebstoffen könnte zu einem ganz neuen Produktionsverfahren im Rahmen von Industrie 4.0 werden“, erläutert Dr. Jörg Tillack, Leiter der 2D-Druckentwicklungen im Geschäftsbereich Lacke,
Klebstoffe, Spezialitäten bei Covestro, vormals Bayer MaterialScience. Der weltweite Inkjet-Markt für „Industrial Printing“ mit einem geschätzten Jahresumsatz von fünf Milliarden Euro erhält mit dem neuen Verfahren die Möglichkeit, am Markt für Industrielacke und Klebstoffe (Jahresumsatz aktuell rund 100 Milliarden Euro) stärker zu partizipieren.

\section{Umfrage zur Digitaltechnologie}

Um die Chancen der Digitaltechnologie besser zu verstehen, hat Covestro in einer Umfrage die Antworten von mehr als 100 Teilnehmern aus der Lack-und Klebstoffindustrie ausgewertet. 93 Prozent von ihnen sind davon überzeugt, dass der Digitaldruck zusätzlichen Wert für ihr Unternehmen schaffen wird. Die Hälfte der befragten Gerätehersteller gab an, dass Kunden bereits heute nach digitalen Lösungen zur Applikation von Lacken und Klebstoffen fragen.

Dank seiner langjährigen Erfahrung versteht Covestro die praktischen Herausforderungen industrieller Lackund Klebstofftechnologien sehr gut. Dabei geht es um weit mehr als um rein dekorative Aspekte: Hinter der geforderten Haltbarkeit und Widerstandsfähigkeit sowie der Adhäsion und Festigkeit von Klebstoffen verbergen sich viele weitere Wünsche, die erfüllt werden müssen.

Das Ergebnis sind oft Formulierungen mit einer relativ hohen Viskosität sowie der Einsatz von Füllstoffen und Pigmenten, die mit industriellem Tintenstrahldruck bisher nicht vereinbar sind. Häufig werden Lacke und Klebstoffe deshalb immer noch in

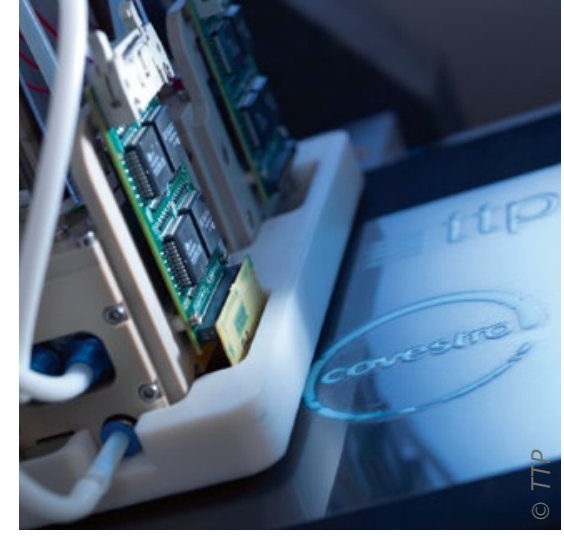

halbautomatischen oder sogar manuellen Auftragsverfahren wie Sprühen oder Streichen eingesetzt. Covestro und TTP arbeiten an Materialsystemen und digitaler Beschichtungstechnologie, um diese Herausforderungen anzugehen und die sich daraus ergebenden Möglichkeiten für die digitale industrielle Applikation von Klebstoffen und Lacken zu erschließen.

\section{Partner gesucht}

Um eine breite Einführung der Technologie $\mathrm{zu}$ fördern, suchen Covestro und TTP nun Partner entlang der Wertschöpfungskette. Beide Unternehmen möchten auch gern interessierte Hersteller von Endprodukten kennenlernen, die den Wunsch haben, Konzepte für Produkte und Prozesse der nächsten Generation mitzuentwickeln. Gefragt sind außerdem Produzenten von Druckköpfen, Druckern und Automatisierungssystemen, die in die Markteinführung dieser Technologie investieren wollen. Anfragen von Entwicklern bei Lack-, Klebstoff- und Tintenherstellern, die Mehrwert für ihre Produkte generieren wollen, sind ebenfalls willkommen. I

\section{Kontakt:}

Covestro AG, Leverkusen, Adam Salmen, Marketingmanager für Digitaldruck, Business Unit Coatings, Adhesives, Specialties, Tel. 021460095727 , adam.salmen@covestro.com www.covestro.com 